\title{
Differences and correlation of serum CEA, CA19-9 and CA72-4 in gastric cancer
}

\author{
JUNXIU YU $^{1}$, SHUGUANG ZHANG ${ }^{2}$ and BINGBO ZHAO ${ }^{1}$ \\ ${ }^{1}$ Department of Gastrointestinal Surgery; ${ }^{2}$ Laboratory Department, \\ Liaocheng People's Hospital and Liaocheng Clinical School of Taishan Medical University, \\ Liaocheng, Shandong 252000, P.R. China
}

Received August 11, 2015; Accepted November 27, 2015

DOI: $10.3892 / \mathrm{mco} .2015 .712$

\begin{abstract}
The sensitivity of three biomarkers, carcinoembryonic antigen (CEA), carbohydrate antigen (CA) 19-9 and CA72-4, in combination has been identified to be greater than that of any of the biomarkers considered in isolation in cases of gastric cancer (GC). However, the fundamental cause underlying this phenomenon remains to be fully elucidated. In the present study, the differences and correlation of these three biomarkers were investigated in patients with GC in order to determine how the three biomarkers in combination work more effectively compared with any of the biomarkers considered alone. The serum levels of CEA, CA19-9 and CA72-4 of 216 patients with gastric adenocarcinoma were analyzed on admission to hospital. The differences in positive rates and the serum levels of CEA, CA19-9 and CA72-4 were analyzed using the $\chi^{2}$ test and the non-parametric Wilcoxon two-sample test. Phi $(\phi)$ correlation analysis was used to study the correlation among the expression (positive or not) levels of CEA, CA19-9 and CA72-4. The correlation among the serum levels of biomarkers was analyzed using Spearman's test. The results demonstrated that the combined positive rate of CEA, CA19-9 and CA72-4 was significantly higher compared with the individual CEA, CA19-9 and CA72-4 positive rates (44.91\% vs. $22.69,18.98$ and $22.69 \%$, respectively; all $\mathrm{P}<0.05$ ). The positive rate of CA19-9 and CA72-4 in the extent of the primary tumor/involvement of regional lymph node/distant metastases (TNM)-III/IV stage subgroup was higher compared with that in the TNM-I/II subgroup $\left(\chi^{2}=5.902, \mathrm{P}=0.015 ; \chi^{2}=8.009\right.$, $\mathrm{P}=0.005)$, although not the positive rate of CEA $\left(\chi^{2}=0.302\right.$, $\mathrm{P}=0.583)$. A significant correlation was identified between the expression level of CEA and CA72-4 ( $\phi$ correlation coefficient $=0.182 ; \mathrm{P}=0.008$ ) and between that of CA19-9 and CA72-4 ( $\phi$ correlation coefficient $=0.189 ; \mathrm{P}=0.006)$, although
\end{abstract}

Correspondence to: Dr Junxiu Yu, Department of Gastrointestinal Surgery, Liaocheng People's Hospital and Liaocheng Clinical School of Taishan Medical University, 67 West Dongchang Road, Liaocheng, Shandong 252000, P.R. China E-mail: yujxcn@hotmail.com

Key words: gastric cancer, tumor marker, CEA, CA19-9, CA72-4 not between that of CEA and CA19-9 ( $\phi$ correlation coefficient=0.048; $\mathrm{P}=0.482$ ) in the total number of patients with $\mathrm{GC}$. A significant correlation was also identified between the serum levels of CEA and CA19-9, of CEA and CA72-4 and of CA19-9 and CA72-4 (Spearman's correlation coefficient $=0.231,-0.271$ and $0.167 ; \mathrm{P}=0.001, \mathrm{P}<0.0001$ and $\mathrm{P}=0.014$, respectively). The present study indicated that there was only a weak correlation between the positive rate of CEA and CA72-4 and between that of CA19-9 and CA72-4, and no correlation was identified between the positive rate of CEA and CA19-9, even if a correlation was identified between the serum levels of the biomarkers. The present study suggested that the evidence that the sensitivity of the three biomarkers in combination is greater than that of any of the biomarkers taken in isolation is due to less co-presentation of CEA, CA19-9 and CA72-4 in patients with GC.

\section{Introduction}

Gastric cancer (GC) is one of most common cancers worldwide, even if the incidence rate is decreasing in certain regions. Almost 951,600 new patients were diagnosed with GC in 2012 (1). The survival rate of patients with GC remains relatively poor, since the majority of cases are already in the late stage at diagnosis, and surgery and chemotherapy result in an unsatisfactory prognosis. Serum tumor markers are useful for diagnosis, for predicting survival rates and for monitoring recurrence following surgery $(2,3)$. Carcinoembryonic antigen (CEA), carbohydrate antigen (CA) 19-9 and CA72-4 are the most frequently used clinical markers. It has been demonstrated that they are useful in the diagnosis, treatment and prognosis of GC (4-8). However, the specificity and sensitivity of serum tumor markers considered in isolation are not satisfactory in GC, particularly in the early stage (9). Therefore, certain researchers have previously used combinations of markers, since they can improve the sensitivity for diagnosis of GC (10-12). Yang et al (13) demonstrated that the sensitivities of CA72-4, CEA, cancer antigen 125 (CA125) and CA19-9 for GC were 33.0, 25.5, 31.1 and 38.7\%, respectively. However, the sensitivity of the four markers in combination increased to $66.0 \%$. Similarly, the individual sensitivities of CEA and CA19-9 were 30 and 42\%, respectively, although this increased to 58\% when CEA and CA19-9 were combined (14). 


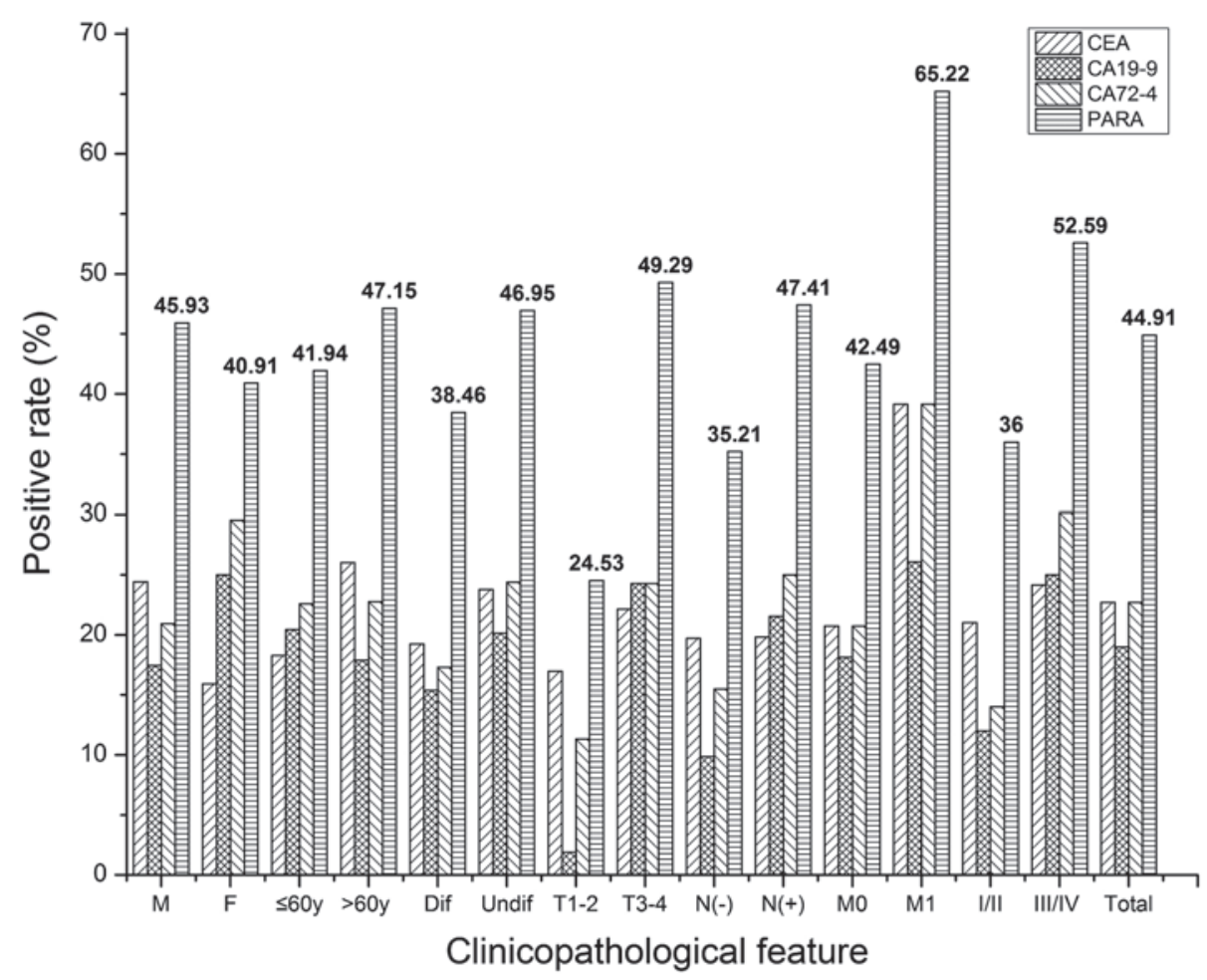

Figure 1. The positive rate of CEA, CA19-9, and CA72-4, and a parallel test of three tumor biomarkers in all patients and in subgroups. M, male; F, female; y, years; Dif, differentiated; Undif, undifferentiated; T, tumor stage; N, lymph node stage; M; M stage; CEA, carcinoembryonic antigen; CA19-9, carbohydrate antigen 19-9; CA72-4, carbohydrate antigen 72-4; PARA, parallel test.

Ychou et al (15) reported a similar result, with a sensitivity of $75 \%$ when CA72-4, CEA and CA19-9 were combined. The above results may be explained simply by different markers being positive in different patients. However, to the best of our knowledge, the fundamental cause underlying this phenomenon remains to be fully elucidated. In the present study, the differences and correlation of these three biomarkers in patients with GC were investigated, with the aim to evaluate how the three biomarkers in combination work more effectively than any of the biomarkers considered alone.

\section{Materials and methods}

Patients. A total of 216 patients from Liaocheng People's Hospital (Shandong, China) were enrolled between January 2011 and December 2013. Of the patients, $172(79.63 \%)$ were men and $44(20.37 \%)$ were women. The median age was 62 years (range: $20-85$ years). The patients' age, gender, tumor histological type and extent of the primary tumor/involvement of regional lymph node/distant metastases (TNM) stage of the cancer were collected from the medical records. The cohort of patients included those who received radical, palliative surgery or chemotherapy. The tumor histological type was determined on the basis of the Japanese Gastric Cancer Association classification system (2010) (16). The T- and the N-stages were not identified in 23 and 29 of the total patients, respectively, since these patients did not receive a radical gastrectomy. These patients were therefore excluded from the statistical comparison performed for the $\mathrm{T}$ - and $\mathrm{N}$-stage groups. Papillary adenocarcinoma and well-/moderately-differentiated tubular adenocarcinoma were classified as differentiated-type carcinoma, whereas poorly differentiated adenocarcinoma, signet ring cell carcinoma and mucinous carcinoma were classified as undifferentiated-type carcinoma. The TNM stage was determined according to the 7th edition of the International Union Against Cancer Classification (2010) (17).

Biomarkers. The serum levels of CEA, CA19-9 and CA72-4 were detected on admission of the patients to hospital, when the patients had not received any surgery, chemotherapy or radiotherapy. The normal reference values were as follows: CEA, $\leq 5 \mathrm{ng} / \mathrm{ml}$; CA19-9, $\leq 39 \mathrm{U} / \mathrm{ml}$; and CA72-4, $\leq 6.9 \mathrm{U} / \mathrm{ml}$. Patients were considered positive for CEA, CA19-9 or CA72-4 when the serum levels were identified to be greater than the above-mentioned values. Patients were considered positive for the parallel test when the serum level of any of CEA, CA19-9 or CA72-4 was greater than its normal value.

Statistical analyses. Differences in the positive rates (categorical variables) of CEA, CA72-4 and CA19-9 were analyzed using the $\chi^{2}$ test, and $\phi$ correlation analysis was used to study the correlation among the expression levels of CEA, CA19-9 and CA72-4. The serum levels of CEA, CA19-9 and CA72-4 were described using median values. The differences in serum levels of the biomarkers were compared using the non-parametric Wilcoxon two-sample test. The correlation between serum levels of biomarkers was analyzed using Spearman's rank correlation coefficient test. All the data were analyzed using SAS version 9.0 software (SAS Institute, Inc., Cary, $\mathrm{NC}$, USA). $\mathrm{P}<0.05$ was considered to indicate a statistically significant difference. 
Table I. The differences among the positive rates of CEA, CA19-9 and CA72-4 for different clinicopathological features in patients with gastric carcinoma.

\begin{tabular}{|c|c|c|c|c|c|c|}
\hline $\begin{array}{l}\text { Clinicopathological } \\
\text { feature }\end{array}$ & $\begin{array}{c}\text { Total } \\
\mathrm{n}\end{array}$ & $\begin{array}{l}\text { CEA } \\
\mathrm{n}(\%)\end{array}$ & $\begin{array}{c}\text { CA19-9 } \\
\text { n }(\%)\end{array}$ & $\begin{array}{r}\text { CA72-4 } \\
\mathrm{n}(\%)\end{array}$ & $\chi^{2}$ & P-value \\
\hline \multicolumn{7}{|l|}{ Gender } \\
\hline Male & 172 & $42(24.42)$ & $30(17.44)$ & $36(20.93)$ & 2.529 & 0.282 \\
\hline Female & 44 & $7(15.91)$ & $11(25.00)$ & $13(29.55)$ & 2.361 & 0.307 \\
\hline$\chi^{2}$ & & 1.447 & 1.302 & 1.483 & & \\
\hline P-value & & 0.229 & 0.254 & 0.223 & & \\
\hline \multicolumn{7}{|l|}{ Age (years) } \\
\hline$\leq 60$ & 93 & 17 (18.28) & $19(20.43)$ & $21(22.58)$ & 0.529 & 0.768 \\
\hline$>60$ & 123 & $32(26.02)$ & 22 (17.89) & $28(22.76)$ & 2.383 & 0.304 \\
\hline$\chi^{2}$ & & 1.807 & 0.223 & 0.001 & & \\
\hline P-value & & 0.179 & 0.637 & 0.975 & & \\
\hline \multicolumn{7}{|l|}{ Histology } \\
\hline Differentiated & 52 & $10(19.23)$ & $8(15.38)$ & $9(17.31)$ & 0.268 & 0.874 \\
\hline Undifferentiated & 164 & 39 (23.78) & $33(20.12)$ & $40(24.39)$ & 0.994 & 0.608 \\
\hline$\chi^{2}$ & & 0.466 & 0.576 & 1.129 & & \\
\hline P-value & & 0.495 & 0.448 & 0.288 & & \\
\hline \multicolumn{7}{|l|}{ T stage } \\
\hline $\mathrm{T} 1-2$ & 53 & $9(16.98)$ & $1(1.89)$ & $6(11.32)$ & 6.810 & 0.033 \\
\hline $\mathrm{T} 3-4$ & 140 & $31(22.14)$ & 34 (24.29) & 34 (24.39) & 0.238 & 0.888 \\
\hline$\chi^{2}$ & & 0.623 & 12.992 & 3.933 & & \\
\hline P-value & & 0.430 & 0.0003 & 0.047 & & \\
\hline \multicolumn{7}{|l|}{$\mathrm{N}$ stage } \\
\hline$N(-)$ & 71 & 14 (19.72) & $7(9.86)$ & $11(15.94)$ & 2.721 & 0.257 \\
\hline $\mathrm{N}(+)$ & 116 & $23(18.93)$ & $25(21.55)$ & $29(25.00)$ & 0.934 & 0.627 \\
\hline$\chi^{2}$ & & 0.0003 & 4.245 & 2.367 & & \\
\hline P-value & & 0.986 & 0.039 & 0.124 & & \\
\hline \multicolumn{7}{|l|}{ M stage } \\
\hline M0 & 193 & $40(20.73)$ & 35 (18.13) & $40(20.73)$ & 0.543 & 0.762 \\
\hline M1 & 23 & $9(39.13)$ & $6(26.09)$ & $9(39.13)$ & 1.150 & 0.563 \\
\hline$\chi^{2}$ & & 3.969 & 0.302 & 3.969 & & \\
\hline P-value & & 0.046 & 0.583 & 0.046 & & \\
\hline \multicolumn{7}{|l|}{ TNM stage ${ }^{a}$} \\
\hline $\mathrm{I} / \mathrm{II}$ & 100 & $21(21.00)$ & $12(12.00)$ & $14(14.00)$ & 3.381 & 0.185 \\
\hline III/IV & 116 & $28(24.14)$ & $29(25.00)$ & $35(30.17)$ & 1.271 & 0.530 \\
\hline$\chi^{2}$ & & 0.302 & 5.902 & 8.009 & & \\
\hline P-value & & 0.583 & 0.015 & 0.005 & & \\
\hline Total cases & 216 & 49 (22.69) & 41 (18.98) & 49 (22.69) & 1.172 & 0.557 \\
\hline
\end{tabular}

Differences in the positive rates (categorical variables) of CEA, CA72-4 and CA19-9 were analyzed using the $\chi^{2}$ test. ${ }^{\mathrm{a} T h e}$ TNM stage was determined according to the 7th edition of the International Union Against Cancer Classification (2010) (17). CEA, carcinoembryonic antigen; CA19-9, carbohydrate antigen 19-9; CA72-4, carbohydrate antigen 72-4.

\section{Results}

Differences and correlation of positive rates of CEA, CA19-9 and $C A 72-4$. The positive rates for CEA, CA19-9 and CA72-4 were 22.69 (49/216), 18.98 (41/216) and 22.69\% (49/216). The positive rate of the parallel test of CEA, CA19-9 and CA72-4 was $44.91 \%$ (97/216), which was significantly higher compared with the individual CEA, CA19-9 and CA72-4 positive rates (all $\mathrm{P}<0.05$; Fig. 1). In subgroups based on gender, age, histology, $\mathrm{T}$ stage, $\mathrm{N}$ stage, $\mathrm{M}$ stage and TNM stage cancer, the majority of the individual positive rates for CEA, CA19-9 and CA72-4 were significantly lower compared with the parallel test positive rates $(\mathrm{P}<0.05)$. However, no significant differences were identified between the parallel test positive rates, the CA19-9 
Table II. Correlation between the expression of CEA, CA19-9 and CA72-4 in patients with gastric carcinoma with respect to different clinicopathological features.

\begin{tabular}{|c|c|c|c|c|c|c|c|c|c|c|}
\hline \multirow[b]{2}{*}{ Clinical feature } & \multirow[b]{2}{*}{$\mathrm{n}$} & \multicolumn{3}{|c|}{ CEA vs. CA19-9 } & \multicolumn{3}{|c|}{ CEA vs. CA72-4 } & \multicolumn{3}{|c|}{ CA19-9 vs. CA72-4 } \\
\hline & & Phi coef & $\chi^{2}$ & P-value & Phi coef & $\chi^{2}$ & P-value & Phi coef & $\chi^{2}$ & P-value \\
\hline \multicolumn{11}{|l|}{ Gender } \\
\hline Male & 172 & -0.012 & 0.023 & 0.879 & 0.173 & 5.166 & 0.023 & 0.140 & 3.378 & 0.066 \\
\hline Female & 44 & 0.323 & & $0.054^{\mathrm{a}}$ & 0.263 & & $0.170^{\mathrm{a}}$ & 0.316 & & $0.057^{\circ}$ \\
\hline \multicolumn{11}{|l|}{ Age } \\
\hline$\leq 60$ years & 93 & 0.174 & & $0.106^{\mathrm{a}}$ & 0.077 & & $0.524^{\mathrm{a}}$ & 0.300 & & $0.011^{\mathrm{a}}$ \\
\hline$>60$ years & 123 & -0.035 & 0.151 & 0.698 & 0.253 & 7.848 & 0.005 & 0.101 & 1.249 & 0.264 \\
\hline \multicolumn{11}{|l|}{ Histology } \\
\hline Diff & 52 & 0.062 & & $0.642^{\mathrm{a}}$ & 0.035 & & $1.000^{\mathrm{a}}$ & 0.228 & & $0.130^{\circ}$ \\
\hline Undiff & 164 & 0.041 & 0.278 & 0.598 & 0.216 & 7.678 & 0.006 & 0.175 & 5.043 & 0.025 \\
\hline \multicolumn{11}{|l|}{ T stage } \\
\hline $\mathrm{T} 1-2$ & 53 & -0.063 & & $1.000^{\mathrm{a}}$ & 0.156 & & $0.267^{\mathrm{a}}$ & 0.388 & & $0.113^{\mathrm{a}}$ \\
\hline T3-4 & 140 & 0.059 & 0.488 & 0.485 & 0.139 & 2.715 & 0.099 & 0.145 & 2.960 & 0.085 \\
\hline $\mathrm{Tx}$ & 23 & -0.071 & & $1.000^{\mathrm{a}}$ & 0.270 & & $0.383^{\mathrm{a}}$ & 0.132 & & $0.643^{2}$ \\
\hline \multicolumn{11}{|l|}{$\mathrm{N}$ stage } \\
\hline $\mathrm{N}(-)$ & 71 & 0.074 & & $0.618^{\mathrm{a}}$ & 0.179 & & $0.209^{\mathrm{a}}$ & -0.011 & & $1.000^{\circ}$ \\
\hline $\mathrm{N}(+)$ & 116 & 0.002 & & $1.000^{\mathrm{a}}$ & 0.162 & 3.055 & 0.081 & 0.278 & 8.991 & 0.003 \\
\hline $\mathrm{Nx}$ & 29 & 0.042 & & $1.000^{\mathrm{a}}$ & 0.193 & & 0.422 & 0.033 & & $1.000^{\circ}$ \\
\hline \multicolumn{11}{|l|}{ M stage } \\
\hline M0 & 193 & 0.058 & 0.648 & 0.421 & 0.149 & 4.170 & 0.039 & 0.109 & 7.014 & 0.008 \\
\hline M1 & 23 & -0.071 & & $1.000^{\mathrm{a}}$ & 0.270 & & $0.383^{\mathrm{a}}$ & 0.132 & & $0.643^{\circ}$ \\
\hline \multicolumn{11}{|l|}{ TNM stage } \\
\hline $\mathrm{I}-\mathrm{II}$ & 100 & 0.036 & & $0.712^{\mathrm{a}}$ & 0.217 & & $0.069^{\mathrm{a}}$ & 0.028 & & $0.674^{\circ}$ \\
\hline III-IV & 116 & 0.047 & 0.251 & 0.616 & 0.156 & 2.819 & 0.093 & 0.228 & 6.015 & 0.014 \\
\hline Total cases & 216 & 0.048 & 0.496 & 0.482 & 0.182 & 7.133 & 0.008 & 0.189 & 7.703 & 0.006 \\
\hline
\end{tabular}

Phi correlation analysis was used to study the correlation among the expression levels of CEA, CA19-9 and CA72-4. 'Fisher's exact test with two-sided P-value. Tx represents a primary tumor that cannot be assessed; Nx represents regional lymph node(s) that cannot be assessed. coef, coefficient; Diff, differentiated; Undiff, undifferentiated; CEA, carcinoembryonic antigen; CA19-9, carbohydrate antigen 19-9; CA72-4, carbohydrate antigen 72-4.

and CA72-4 positive rates in the women patients, or between the parallel test positive rates and the CEA and CA72-4 positive rates in the $\mathrm{T} 1 / 2$ and $\mathrm{M} 1$ subgroups $(\mathrm{P}>0.05)$.

As shown in Table I, the positive rate of CEA, CA19-9 or CA72-4 did not differ significantly in all 216 patients. In the subgroups, no significant difference was identified in the positive rate of CEA, CA19-9 and CA72-4, with the exception of the T1/2-stage patients $\left(\chi^{2}=6.810, \mathrm{P}=0.033\right)$.

The differences in the positive rates of CEA, CA19-9 and CA72-4 according to gender, age, histology, $\mathrm{T}$ stage, $\mathrm{N}$ stage, $M$ stage and TNM stage in each subgroup were analyzed using the $\chi^{2}$ test. The positive rate of CEA in the M1 subgroup was higher compared with that in the M0 subgroup. No differences in the positive rate of CEA were identified on the basis of gender, age, histology, $\mathrm{T}$ stage, $\mathrm{N}$ stage or the TNM stage. The positive rate of CA19-9 in the T3/4, N(+) and TNM-III/IV stage subgroups was higher compared with that in the T1/2, N(-) and TNM-I/II stage subgroups. No differences in the positive rate of CA19-9 were identified according to other clinicopatho- logical classifications. The positive rate of CA72-4 in the T3/4, M1 and TNM-III/IV stage subgroups was higher compared with that in the T1/2, M0 and TNM-I/II stage subgroups (Table I).

The phi $(\phi)$ correlation analysis demonstrated that no significant correlation existed between the expression levels (positive or not) of CEA and CA19-9 in all 216 patients. However, a significant correlation did exist between the expression levels of CEA and CA72-4, and between that of CA19-9 and CA72-4, in all cases. In all the subgroups, no significant correlation was identified between CEA and CA19-9. However, a significant correlation was identified between the expression level of CEA and CA72-4 in men, in patients aged $>60$ years, in patients exhibiting an undifferentiated histology and in the M0 subgroup of GC, although not in other subgroups. The majority of the $\phi$ correlations between the expression levels of CA19-9 and CA72-4 were significant in patients aged $\leq 60$ years, and in those with undifferentiated histology or N(+), M0 or TNM-III/IV stage cancer (Table II). 


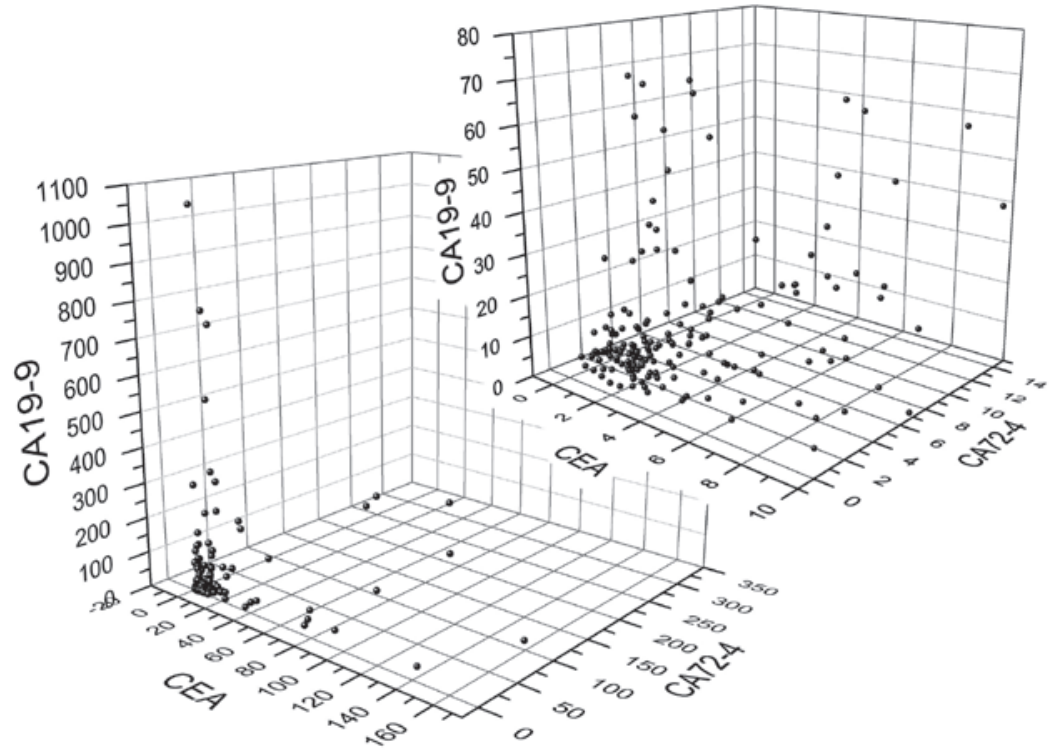

Figure 2. Three-dimensional scatter plot of the serum levels of CEA, CA19-9 and CA72-4. The left lower graph includes all the data, and the right upper graph includes a portion of the data (CEA, $\leq 10 \mathrm{ng} / \mathrm{ml}$; CA19-9, $\leq 80 \mathrm{U} / \mathrm{ml}$; and CA72-4, $\leq 14 \mathrm{U} / \mathrm{ml})$. CEA, carcinoembryonic antigen; CA19-9, carbohydrate antigen 19-9; CA72-4, carbohydrate antigen 72-4.

Differences and correlation of the serum levels of CEA, CA19-9 and CA72-4. The median serum levels of CEA, CA19-9 and CA72-4 were 2.230 (range, 0.2-152.7) ng/ml, 7.50 (range, $0-1057.9$ ) $\mathrm{U} / \mathrm{ml}$ and 2.51 (range, 0.503-300.0) $\mathrm{U} / \mathrm{ml}$, respectively. The three-dimensional scatter plot is shown in Fig. 2.

The Wilcoxon two-sample test revealed that the serum levels of CEA differed significantly, according to the gender. The serum level of CEA was higher in women compared with men (2.465 vs. $1.175 \mathrm{ng} / \mathrm{ml})$. No significant differences were identified in the serum level of CEA according to age, histology, or the T, N, M or TNM stages of cancer. The serum levels of CA19-9 in the T3/4 and the TNM-III/IV stages were significantly higher compared with those in the T1/2 and the TNM-I/II stages, respectively. The serum level of CA72-4 in the T3/4, M1 and TNM-III/IV stage subgroups was significantly higher than in the T1/2, M0 and TNM-I/II stage subgroups. No significant differences were identified in the serum level of CA19-9 and CA72-4 in the other clinicopathological subgroups. These results are shown in Table III.

Spearman's correlation analysis showed that there were significant correlations between the serum levels of CEA and CA19-9, of CEA and CA72-4, and of CA19-9 and CA72-4 in all 216 patients (Table IV). Regarding the subgroups, there were significant correlations between the serum levels of CEA and CA19-9 in men, women, patients aged $>60$ years, and those with differentiated tumors, undifferentiated tumors, and T3/4, N(+), M0, TNM-I/II or TNM-III/IV stage cancer, but not in patients aged $\leq 60$ years and those with $\mathrm{T} 1 / 2$, $\mathrm{N}(-)$ or M1 stage cancer. In the majority of subgroups, there were significant correlations between the serum levels of CEA and CA72-4, except in the cases of T1/2, N(-) and M1 stage cancer. A significant correlation was identified between the serum levels of CA19-9 and CA72-4 in women patients, those aged $\leq 60$ years, those with undifferentiated tumors and those with $\mathrm{N}(+), \mathrm{M} 0$ and TNM-III/IV stage cancer, although not in the other subgroups.

\section{Discussion}

CEA was originally described by Gold and Freedman in 1965 (18); it is a glycoprotein that belongs to the immunoglobulin superfamily. CEA is used predominantly for the management of colorectal carcinoma, and its levels may be increased in gastric, lung, pancreatic and breast carcinoma. CA19-9 was first isolated by Koprowski et al (19) in 1979; it is a high-molecular-mass mucin glycoprotein complex that is associated with the Lewis A blood group. CA19-9 is used primarily as a tumor marker for pancreatic cancer, although it has been used as a tumor marker in other gastrointestinal cancers, including colorectal and gastric carcinoma, and gallbladder cancer. It may also be increased in several benign diseases, including pancreatitis and cholelithiasis. CA72-4 was first described by Colcher et al (20) in 1981: It is a glycoprotein with a molecular mass $>1000 \mathrm{kDa}$ and is associated with the sialylated Lewis Tn group. It is a tumor marker for numerous cancers, including breast, ovarian, colorectal and pancreatic cancer, and it has good specificity for GC.

No significant differences were identified in the positive rate of CEA, CA19-9 and CA72-4 in all 216 patients, and almost all the subgroups, in the present study. Only $20 \%$ of all the patients with GC exhibited positive rates of CEA, CA19-9 and CA72-4. The results in the present study were similar to those previously obtained: In a systematic review (9) of 187 publications with data for CEA and CA19-9, and 19 publications with data for CEA, CA72-4 and CA19-9, the positive rates were $21.1 \%$ for CEA, $27.8 \%$ for CA19-9 and $30.0 \%$ for CA72-4. In a study of 149 Chinese patients with GC, He et al (10) demonstrated that the positive rates for CEA and CA19-9 were 17.4 and $20.8 \%$, respectively, which were not significantly different. In a subsequent study of Chinese patients with GC, the preoperative serum positive rate of CEA was $24.0 \%$, which was similar to the serum positive rate of CA19-9 (18.9\%) (21). Mittal et al (22) demonstrated that the positive rate of CEA 
Table III. Comparison of the serum levels of CEA, CA19-9 and CA72-4 with respect to different clinicopathological features in patients with gastric carcinoma.

\begin{tabular}{|c|c|c|c|c|c|c|c|c|c|c|}
\hline \multirow[b]{2}{*}{ ClinPath feature } & \multirow[b]{2}{*}{$\mathrm{n}$} & \multicolumn{3}{|c|}{ CEA } & \multicolumn{3}{|c|}{ CA19-9 } & \multicolumn{3}{|c|}{ CA72-4 } \\
\hline & & Median & $\mathrm{Z}$ & P-value & Median & $\mathrm{Z}$ & P-value & Median & $\mathrm{Z}$ & P-value \\
\hline Gender & & & -4.005 & $<0.001$ & & 0.768 & 0.443 & & 0.450 & 0.653 \\
\hline Male & 172 & 2.465 & & & 7.450 & & & 2.510 & & \\
\hline Female & 44 & 1.175 & & & 12.000 & & & 2.575 & & \\
\hline Age (years) & & & -0.440 & 0.660 & & 0.459 & 0.647 & & 0.001 & 0.999 \\
\hline$\leq 60$ & 93 & 2.310 & & & 7.500 & & & 2.820 & & \\
\hline$>60$ & 123 & 2.230 & & & 7.500 & & & 2.390 & & \\
\hline Histology & & & -0.088 & 0.930 & & -0.693 & 0.489 & & -1.483 & 0.138 \\
\hline Diff & 52 & 2.270 & & & 7.450 & & & 1.970 & & \\
\hline Undiff & 164 & 2.220 & & & 7.700 & & & 2.810 & & \\
\hline T stage & & & -0.289 & 0.773 & & -2.376 & 0.018 & & -3.241 & 0.001 \\
\hline $\mathrm{T} 1-2$ & 53 & 2.230 & & & 6.600 & & & 1.530 & & \\
\hline T3-4 & 140 & 2.170 & & & 9.700 & & & 2.745 & & \\
\hline $\mathrm{N}$ stage & & & 0.860 & 0.390 & & -1.607 & 0.108 & & -1.868 & 0.062 \\
\hline $\mathrm{N}(-)$ & 71 & 2.230 & & & 6.600 & & & 1.880 & & \\
\hline $\mathrm{N}(+)$ & 116 & 2.075 & & & 8.775 & & & 2.685 & & \\
\hline M stage & & & 1.698 & 0.090 & & 0.579 & 0.563 & & 2.072 & 0.038 \\
\hline M0 & 193 & 2.210 & & & 7.400 & & & 2.320 & & \\
\hline M1 & 23 & 4.130 & & & 11.200 & & & 6.290 & & \\
\hline TNM stage & & & -0.011 & 0.991 & & -2.721 & 0.007 & & -3.883 & 0.0001 \\
\hline $\mathrm{I} / \mathrm{II}$ & 100 & 2.370 & & & 6.600 & & & 1.795 & & \\
\hline III/IV & 116 & 2.115 & & & 11.000 & & & 3.155 & & \\
\hline
\end{tabular}

The non-parametric Wilcoxon two-sample test was used for the comparison of the differences in serum levels of the biomarkers. ${ }^{\text {aTwo-sided }}$ P-values. ClinPath, clinicopathological; Diff, differentiated; Undiff, undifferentiated; CEA, carcinoembryonic antigen; CA19-9, carbohydrate antigen 19-9; CA72-4, carbohydrate antigen 72-4.

was lower compared with that of CA19-9, although no significant differences were identified between the positive rate of CEA and CA72-4, or of CA19-9 and CA72-4. In previous studies $(6,23)$, the positive rate of CEA was determined to be lower compared with that of CA19-9 and CA72-4.

The positive rate of the parallel test for CEA, CA19-9 and CA72-4 was significantly higher compared with the individual positive rates for CEA, CA19-9 and CA72-4 in all 216 patients. In the subgroups, the majority of the parallel test positive rates were also higher compared with the individual CEA, CA19-9, and CA72-4 positive rates.

In the gender, age and histological subgroups, no significant differences were identified in the positive rate of CEA, CA19-9 and CA72-4. The serum levels of CEA, CA19-9 and CA72-4 did not differ significantly on the basis of gender, age or histological classification, with the exception of the serum level of CEA, which differed between the genders. The results of the present study were supported by those of previous studies. No significant differences were identified in the positive rate of CEA, CA19-9 and CA72-4 between men and women, between patients aged $<60$ and $\geq 60$ years, or among the various histological types (24). Ucar et al (25) demonstrated that no significant differences existed among the positive rates of
CEA, CA19-9 and CA72-4, according to gender or the histological type (intestinal or diffuse). Choi et al (8) demonstrated that the positive rate of CA19-9 was not correlated with gender and age, although it was correlated with histological type. Yajima et al (26) identified that the serum levels of CA19-9 did not correlate with gender, age or histological type.

The positive rate of CEA did not increase in association with the progression of GC. The significant difference of the positive rate of CEA was presented only in the M subgroup. No significant differences were identified in the positive rates of CEA according to the T, N or TNM stages of cancer in the present study. These results are supported by previous studies: Cidon et al (23) demonstrated that the positive rate of CEA in early-stage GC did not differ from that in advanced-stage GC. Ucar et al (25) identified that the positive rate did not correlate with the TNM stage of cancer, serosal involvement or the $\mathrm{N}$ stage, although it was correlated with liver involvement. However, a number of previous studies indicated that the positive rate of CEA does increase with tumor progression. In a systematic review, Shimada et al (9) revealed that the positive rate of serum CEA was significantly correlated with the TNM stage. Tian et al (27) demonstrated that there were significant differences in the positive rate of serum CEA between 
Table IV. The correlation between serum levels of CEA, CA19-9, CA72-4 in patients with gastric carcinoma in different clinicopathological features.

\begin{tabular}{|c|c|c|c|c|c|c|c|}
\hline \multirow[b]{2}{*}{ ClinPath feature } & \multirow[b]{2}{*}{$\mathrm{N}$} & \multicolumn{2}{|c|}{ CEA vs. CA19-9 } & \multicolumn{2}{|c|}{ CEA vs. CA72-4 } & \multicolumn{2}{|c|}{ CA19-9 vs. CA72-4 } \\
\hline & & Coef & P-value & Coef & P-value & Coef & P-value \\
\hline \multicolumn{8}{|l|}{ Gender } \\
\hline Male & 172 & 0.217 & 0.004 & 0.266 & 0.000 & 0.065 & 0.399 \\
\hline Female & 44 & 0.376 & 0.012 & 0.330 & 0.029 & 0.465 & 0.002 \\
\hline \multicolumn{8}{|l|}{ Age } \\
\hline$\leq 60$ years & 93 & 0.186 & 0.075 & 0.238 & 0.022 & 0.299 & 0.004 \\
\hline$>60$ years & 123 & 0.263 & 0.003 & 0.313 & 0.000 & 0.060 & 0.513 \\
\hline \multicolumn{8}{|l|}{ Histology } \\
\hline Differ & 52 & 0.285 & 0.041 & 0.342 & 0.013 & 0.018 & 0.901 \\
\hline Undiffer & 164 & 0.213 & 0.006 & 0.266 & 0.001 & 0.211 & 0.007 \\
\hline \multicolumn{8}{|l|}{ T-stage } \\
\hline $\mathrm{T} 1-2$ & 53 & 0.219 & 0.116 & 0.153 & 0.274 & -0.020 & 0.889 \\
\hline T3-4 & 140 & 0.255 & 0.002 & 0.294 & 0.000 & 0.162 & 0.056 \\
\hline \multicolumn{8}{|l|}{ N-stage } \\
\hline $\mathrm{N}(-)$ & 71 & 0.207 & 0.083 & 0.112 & 0.354 & -0.057 & 0.640 \\
\hline $\mathrm{N}(+)$ & 116 & 0.267 & 0.004 & 0.347 & 0.000 & 0.300 & 0.001 \\
\hline \multicolumn{8}{|l|}{ M-stage } \\
\hline M0 & 193 & 0.251 & 0.000 & 0.255 & 0.000 & 0.174 & 0.015 \\
\hline M1 & 23 & 0.022 & 0.920 & 0.261 & 0.229 & 0.089 & 0.688 \\
\hline \multicolumn{8}{|l|}{ TNM-stage } \\
\hline $\mathrm{I} / \mathrm{II}$ & 100 & 0.211 & 0.036 & 0.297 & 0.002 & -0.046 & 0.646 \\
\hline III/IV & 116 & 0.234 & 0.011 & 0.288 & 0.001 & 0.239 & 0.010 \\
\hline Total cases & 216 & 0.231 & 0.001 & -0.271 & $<.0001$ & 0.167 & 0.014 \\
\hline
\end{tabular}

Coef, Spearman's correlation coefficient; ClinPath, clinicopathological; Diff, differentiated; Undiff, undifferentiated; CEA, carcinoembryonic antigen; CA19-9, carbohydrate antigen 19-9; CA72-4, carbohydrate antigen 72-4.

the T1/2 and T3/4 stages, and between cases with lymph node metastasis and those with no lymph node metastasis.

The positive rate of CA19-9 increased with the progression of GC. The positive rate of CA19-9 in the T3/4, N(+) and TNM-III/IV subgroups was higher compared with that in the T1/2, N(-) and TNM-I/II subgroups. Shimada et al (9) presented similar results on the basis of a systematic review, which identified that the positive rate of CA19-9 increased with the TNM stage. These results indicated that CA19-9 is an improved marker for GC prognosis (28). Lee et al (11) identified that the positivity of CA19-9 increased significantly with GC progression, similarly to the results in the present study. The positivity of CA19-9 was $4.0 \%$ in stage I, $6.8 \%$ in stage II, $13.9 \%$ in stage III and $24.1 \%$ in stage IV GC. A previous study (21) revealed that the positive rate of CA19-9 was not correlated with the T, M or TNM-stage GC cases. Another study demonstrated that there were significant increases in the level of CA19-9 in patients with GC, with direct invasion and extensive peritoneal seeding (29).

The positive rate of CA72-4 increased in accordance with the progression of GC. The positive rate of CA72-4 in the T3/4, M1 and TNM-III/IV subgroups was higher compared with that in the T1/2, M0 and TNM-I/II subgroups. A systematic review by Shimada et al (9) indicated that the positive rate of CA72-4 increased with the TNM stage. The specificity of CA72-4 for GC is higher compared with that of CEA and CA19-9. A meta-analysis of Chinese patients with GC (30) demonstrated that CA72-4 was selected as the preferable single test, with the highest accumulated accuracy among tumor markers, including CA72-4, CA12-5, CA15-3, CA19-9, CA24-2 and CEA. The CA72-4 was identified as a tumor marker for late-stage GC in previous studies. Emoto et al (31) determined that the sensitivity of CA72-4 for the peritoneal metastasis of GC was $45 \%$, which was higher compared with that of CEA and CA19-9. Patients with ovarian metastasis exhibited significantly higher levels of CA72-4 (31).

The serum levels of CA19-9 and CA72-4 increased with tumor progression, although the serum level of CEA did not. CEA was revealed not to be higher in the T3/4, N(+), M1 and TNM-III/IV stages, compared with the T1/2, N(-), M0 and TNM-I/II stages. The serum level of CA19-9 was higher in the T3/4 and TNM-III/IV stages compared with the T1/2 and the TNM-I/II stages. The serum level of CA72-4 was higher in the T3/4, M1 and TNM-III/IV stages compared with the T1/2, M0 and TNM-I/II stages. Subsequently to the aforementioned results, the serum level of CA19-9 and 
CA72-4 in the TNM-III/IV stage was higher compared with the TNM-I/II stage, although there were conflicting results for the difference in the serum level of CA19-9 and CA72-4 in the T, N and M stages. Han et al (29) identified that the mean values of CEA did not differ significantly among the serosa-unexposed, serosa-exposed, direct invasion and localized peritoneal seeding groups. The mean CEA level was higher in patients with extensive peritoneal seeding. Yajima et al (26) identified that the patients with higher serum levels of CA19-9 presented with advanced T stage, $\mathrm{N}$ stage and TNM stage cancer. However, Lee et al (11) revealed that there was a tendency towards increasing CEA levels with advancing stages of cancer, as well as increases in CA19-9 and CA72-4.

In all the patients, the $\phi$ correlation analysis indicated that there was significant correlation between the expression of CEA and CA72-4, and between CA72-4 and CA19-9. The correlation coefficients between CEA and CA72-4 and between CA72-4 and CA19-9 was $<0.2$, indicating a weak correlation. No significant correlation was identified between CEA and CA19-9. However, in certain of the subgroups, a significant correlation was identified between the expression of CEA and CA72-4, and between CA19-9 and CA72-4. The results in the present study revealed that CEA, CA19-9 and CA72-4 did not exhibit similar trends in positivity. Statistical analyses revealed that different patients presented with different biomarkers, even if the positive rate was determined to be similar among the three biomarkers.

In all 216 patients, a significant correlation was identified between the serum levels of CEA and CA19-9, of CEA and CA72-4, and of CA19-9 and CA72-4. In the T1/2 stage subgroup, no significant correlations between the serum levels of CEA and CA19-9, of CEA and CA72-4, and of CA19-9 and CA72-4 were identified. In the T3/4 stage subgroup, a significant correlation was identified between the serum level of CEA and CA19-9, and also between that of CEA and CA72-4, and an almost significant correlation existed between CA19-9 and CA72-4 $(\mathrm{P}=0.056)$. In the $\mathrm{N}$ stage subgroup, a significant correlation was identified between the serum levels of CEA and CA19-9, of CEA and CA72-4, and of CA19-9 and CA72-4 in $\mathrm{N}(+)$ stage patients, although not in $\mathrm{N}(-)$ stage patients. These results indicated that a significant correlation was identified between the serum levels of CEA, CA19-9 and CA72-4 in the late $\mathrm{T}$ and $\mathrm{N}$ stages, although not in the early $\mathrm{T}$ and $\mathrm{N}$ stages. However, the opposite was shown for the M stage. A significant correlation was identified between the serum levels of CEA, CA19-9 and CA72-4 in the M0 stage, although not in the M1 stage. There have been few studies published on the correlation among biomarkers. Han et al (29) obtained different results: Significant correlations between the serum levels of CEA and CA19-9 were identified in their laboratory for most patients, including the serosa-unexposed group (although not the serosa-exposed group), and patients with direct invasion, localized peritoneal seeding and extensive peritoneal seeding.

In conclusion, the present study identified only a weak correlation between the positive rates of CEA and CA72-4, and of CA19-9 and CA72-4, and no correlation was identified between the positive rate of CEA and CA19-9 in all the patients with GC, even if there was a marked correlation between the serum levels of CEA and CA19-9, CA19-9 and CA72-4, and CA19-9 and CA72-4. In addition, the positive rate and serum level of CEA did not increase with tumor progression, although the positive rate and levels of CA19-9 and CA72-4 did increase with tumor progression. These results indicated that different biomarkers presented positive in different cases and during different stages of GC. There was less co-presentation of CEA, CA19-9 and CA72-4 in patients with GC. Therefore, the combined value of the tests, particularly of CA19-9 and CA72-4, is complementary. The sensitivity of the three biomarkers in combination is greater than that of any of the biomarkers taken in isolation.

\section{Acknowledgements}

We would like to thank all members of the staff in the Department of Gastrointestinal Surgery, Department of Pathology and the Medical Record Department for their support in data accumulation.

\section{References}

1. Torre LA, Bray F, Siegel RL, Ferlay J, Lortet-Tieulent J and Jemal A: Global cancer statistics, 2012. CA Cancer J Clin 65: 87-108, 2015.

2. Park HJ, Ahn JY, Jung HY, Lim H, Lee JH, Choi KS, Kim do H, Choi KD, Song HJ, Lee GH and Kim JH: Clinical characteristics and outcomes for gastric cancer patients aged 18-30 years. Gastric Cancer 17: 649-660, 2014.

3. Nam DH, Lee YK, Park JC, Lee H, Shin SK, Lee SK, Lee YC, Cheong JH, Hyung WJ, Noh SH and Kim CB: Prognostic value of early postoperative tumor marker response in gastric cancer. Ann Surg Oncol 20: 3905-3911, 2013.

4. Mohri Y, Tanaka K, Ohi M, Saigusa S, Yasuda H, Toiyama Y, Araki T, Inoue Y and Kusunoki M: Identification of prognostic factors and surgical indications for metastatic gastric cancer. BMC Cancer 14: 409, 2014.

5. Kwon OK, Yu W and Chung H: Prognostic value of postoperative CA19-9 normalization in patients with advanced gastric cancer. Hepatogastroenterology 60: 240-243, 2013.

6. Kim DH, Oh SJ, Oh CA, Choi MG, Noh JH, Sohn TS, Bae JM and Kim S: The relationships between perioperative CEA, CA 19-9, and CA 72-4 and recurrence in gastric cancer patients after curative radical gastrectomy. J Surg Oncol 104: 585-591, 2011.

7. Jo JC, Ryu MH, Koo DH, Ryoo BY, Kim HJ, Kim TW, Choi KD, Lee GH, Jung HY, Yook JH, et al: Serum CA 19-9 as a prognostic factor in patients with metastatic gastric cancer. Asia Pac J Clin Oncol 9: 324-330, 2013

8. Choi AR, Park JC, Kim JH, Shin SK, Lee SK, Lee YC and Chung JB: High level of preoperative carbohydrate antigen 19-9 is a poor survival predictor in gastric cancer. World J Gastroenterol 19: 5302-5308, 2013.

9. Shimada H, Noie T, Ohashi M, Oba K and Takahashi Y: Clinical significance of serum tumor markers for gastric cancer: A systematic review of literature by the task force of the Japanese gastric cancer association. Gastric Cancer 17: 26-33, 2014.

10. He CZ, Zhang KH, Li Q, Liu XH, Hong Y and Lv NH: Combined use of AFP, CEA, CA125 and CA19-9 improves the sensitivity for the diagnosis of gastric cancer. BMC Gastroenterol 13: 87, 2013.

11. Lee JC, Lee SY, Kim CY and Yang DH: Clinical utility of tumor marker cutoff ratio and a combination scoring system of preoperative carcinoembryonic antigen, carbohydrate antigen 19-9, carbohydrate antigen 72-4 levels in gastric cancer. J Korean Surg Soc 85: 283-289, 2013.

12. Li F, Li S, Wei L, Liang X, Zhang H and Liu J: The correlation between pre-operative serum tumor markers and lymph node metastasis in gastric cancer patients undergoing curative treatment. Biomarkers 18: 632-637, 2013.

13. Yang AP, Liu J, Lei HY, Zhang QW, Zhao L and Yang GH: CA72-4 combined with CEA, CA125 and CA19-9 improves the sensitivity for the early diagnosis of gastric cancer. Clin Chim Acta 437: 183-186, 2014.

14. Bagaria B, Sood S, Sharma R and Lalwani S: Comparative study of CEA and CA19-9 in esophageal, gastric and colon cancers individually and in combination (ROC curve analysis). Cancer Biol Med 10: 148-157, 2013. 
15. Ychou M, Duffour J, Kramar A, Gourgou S and Grenier J: Clinical significance and prognostic value of CA72-4 compared with CEA and CA19-9 in patients with gastric cancer. Dis Markers 16: 105-110, 2000

16. Japanese Gastric Cancer Association: Japanese classification of gastric carcinoma: 3rd English edition. Gastric Cancer 14: 101-112, 2011.

17. Edge SB, Byrd DR, Compton CC, Fritz AG, Greene FL and Trotti A: AJCC Cancer Staging Handbook. Springer, New York, 2010.

18. Gold P and Freedman SO: Demonstration of tumor-specific antigens in human colonic carcinomata by immunological tolerance and absorption techniques. J Exp Med 121: 439-462, 1965.

19. Koprowski H, Steplewski Z, Mitchell K, Herlyn M, Herlyn D and Fuhrer P: Colorectal carcinoma antigens detected by hybridoma antibodies. Somatic Cell Genet 5: 957-971, 1979.

20. Colcher D, Hand PH, Nuti M and Schlom J: A spectrum of monoclonal antibodies reactive with human mammary tumor cells. Proc Natl Acad Sci USA 78: 3199-3203, 1981

21. Huang ZB, Zhou X, Xu J, Du YP, Zhu W, Wang J, Shu YQ and Liu P: Prognostic value of preoperative serum tumor markers in gastric cancer. World J Clin Oncol 5: 170-176, 2014.

22. Mittal A, Gupta SP, Jha DK, Sathian B and Poudel B: Impact of various tumor markers in prognosis of gastric cancer. A hospital based study from tertiary care hospital of Kathmandu valley. Asian Pac J Cancer Prev 14: 1965-1967, 2013.

23. Cidón EU and Bustamante R: Gastric cancer: Tumor markers as predictive factors for preoperative staging. J Gastrointest Cancer 42: 127-130, 2011
24. Liu X, Cai H and Wang Y: Prognostic significance of tumour markers in Chinese patients with gastric cancer. ANZ J Surg 84: 448-453, 2014

25. Ucar E, Semerci E, Ustun H, Yetim T, Huzmeli C and Gullu M: Prognostic value of preoperative CEA, CA 19-9, CA 72-4, and AFP levels in gastric cancer. Adv Ther 25: 1075-1084, 2008.

26. Yajima H, Omura N, Matai K, Mitsumori N, Yoshida K and Yanaga K: Clinicopathological features of CA19-9-producing gastric cancer. Hepatogastroenterology 61: 221-225, 2014.

27. Tian SB, Yu JC, Kang WM, Ma ZQ, Ye X, Cao ZJ and Yan C: Combined detection of CEA, CA 19-9, CA 242 and CA 50 in the diagnosis and prognosis of resectable gastric cancer. Asian Pac J Cancer Prev 15: 6295-6300, 2014.

28. Sisik A, Kaya M, Bas G, Basak F and Alimoglu O: CEA and CA 19-9 are still valuable markers for the prognosis of colorectal and gastric cancer patients. Asian Pac J Cancer Prev 14: 4289-4294, 2013.

29. Han ES, Lee HH, Lee JS, Song KY, Park CH and Jeon HM: At which stage of gastric cancer progression do levels of carcinoembryonic antigen and carbohydrate antigen 19-9 increase? Application in advanced gastric cancer treatment. J Gastric Cancer 14: 123-128, 2014.

30. Chen XZ, Zhang WK, Yang K, Wang LL, Liu J, Wang L, Hu JK, Zhang B, Chen ZX, Chen JP, et al: Correlation between serum CA724 and gastric cancer: Multiple analyses based on Chinese population. Mol Biol Rep 39: 9031-9039, 2012.

31. Emoto S, Ishigami H, Yamashita H, Yamaguchi H, Kaisaki S and Kitayama J: Clinical significance of CA125 and CA72-4 in gastric cancer with peritoneal dissemination. Gastric Cancer 15: 154-161, 2012. 